\title{
Spreading of Slip from a Region of Low Friction
}

\author{
By \\ Maria Comninou, Ann Arbor, Michigan, and J. Dundurs, Evanston, Illinois \\ With 3 Figures
}

(Received February 8, 1982)

\section{Summary}

Recent models of earthquake faults involve heterogeneous slip regions along the faults. Some of this work suggests the following problem: two solids of different material properties are pressed together and sheared. Then, slip propagates asymmetrically from a region of low friction.

\section{Introduction}

Current thinking on earthquake faults maintains that the behavior of slip along fault planes is heterogeneous [1]. Thus, regions of higher resistance to slip (asperity model) are postulated, or, alternatively, regions on which slip cannot occur at all are presumed (barrier model). The analytical work associated with these geophysical models has been largely confined to the application of solutions already available in the literature for collinear cracks. These solutions have the major drawback that they involve singular shear tractions at the transitions from slip to no slip (stick) regions, and are of limited use. In this paper we consider specifically the problem of slip in the presence of a non-uniform frictional law, but otherwise we opt for simplicity. We thus take the fault plane at the interface of two unbounded solids of different material properties. The solids are compressed and sheared at infinity with the uniform tractions $p^{\infty}$ and $q^{\infty}$, as shown in Fig. 1.

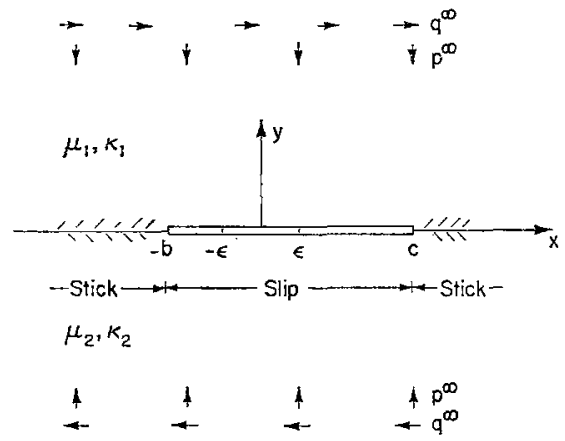

Fig. 1. Geometry of the problem 
We assume that Coulomb's law of friction applies but that the coefficient of friction $f$ varies along the fault plane, or $f=f(x)$. We are interested in studying the effect of sudden drop in friction, and we take $f(x)$ to be a constant $f+\Delta f$ everywhere except in the region $|x|<\varepsilon$ where it drops parabolically to a minimum value $f$ at $x=0$, Fig. 2 . Thus the maximum drop is $\Delta f$. To avoid catastrophic slip we consider a combination of applied tractions such that

$$
f p^{\infty}<q^{\infty}<(f+\Delta f) p^{\infty} .
$$

Slip must then occur in a region surrounding the origin. The extent of the slip zone is, however, unknown.

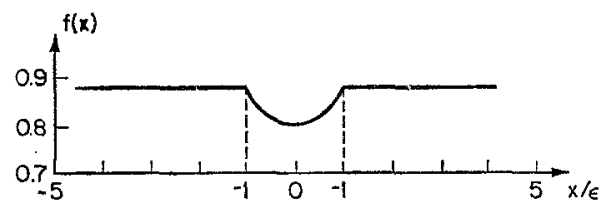

Fig. 2. Coefficient of friction $f(x)$

\section{Formulation}

We model the slip by a distribution of glide dislocations with density $B_{x}(x)$. If the extent of slip is determined by the as yet unknown parameters $b$ and $c$, Fig. 1, then the normal and shear tractions transmitted by the interface can be obtained as in [2]. They are

$$
\begin{aligned}
& N(x)=-p^{\infty}+\beta B_{x}(x), \\
& S(x)=q^{\infty}-\frac{C}{\pi} \int_{-b}^{e} \frac{B_{x}(\xi)}{\xi-x} d \xi,
\end{aligned}
$$

where

$$
\begin{aligned}
& C=\frac{2 \mu_{1}(1+\alpha)}{\left(\varkappa_{1}+1\right)\left(1-\beta^{2}\right)}, \\
& \alpha=\frac{\mu_{2}\left(\varkappa_{1}+1\right)-\mu_{1}\left(\varkappa_{2}+1\right)}{\mu_{2}\left(\varkappa_{1}+1\right)+\mu_{1}\left(\varkappa_{2}+1\right)} \\
& \beta=\frac{\mu_{2}\left(\varkappa_{1}-1\right)-\mu_{1}\left(\varkappa_{2}-1\right)}{\mu_{2}\left(\varkappa_{1}+1\right)+\mu_{1}\left(\varkappa_{2}+1\right)} .
\end{aligned}
$$

This formulation ensures continuity of tractions at the interface as well as continuity of normal displacements. The only boundary condition to be satisfied is then

$$
S(x)=-f(x) N(x) \quad-b<x<c
$$

where

$$
f(x)=f\left\{1+\frac{\Delta f}{f}\left[\left(1-\frac{x^{2}}{\varepsilon^{2}}\right) H\left(\varepsilon^{2}-x^{2}\right)\right]\right\}
$$


is the variable coefficient of friction, Fig. 2, as described in the Introduction, and $H($ ) denotes the Heaviside step function. Moreover, since separation is neither anticipated nor allowed in the present formulation, we assume that

$$
N(x) \leqq 0 \quad-\infty<x<\infty
$$

and

$$
|S(x)|<f(x)|N(x)| \quad x>c, \quad x<-b
$$

outside the slip zone. These inequalities must be verified a posteriori.

Using (2) and (3), (7) becomes

$$
-\beta f(x) B_{x}(x)+\frac{1}{\pi} \int_{-b}^{c} \frac{B_{x}(\xi)}{\xi-x} d \xi=\frac{1}{C}\left[q^{\infty}-p^{\infty}\right] f(x), \quad-b<x<c
$$

where $B_{x}(x)$ must be bounded for bounded shear tractions. In addition we must require

$$
\int_{-b}^{c} B_{x}(\xi) d \xi=0
$$

for single-valued displacements. Equation (11) is a Cauchy singular integral equation of the second kind with variable coefficients. Its general solution is given by Tricomi [3]. A bounded solution is possible provided that a certain consistency condition [4] involving the characteristic funicton and the right side of (11) is satisfied. In such case, the solution can be written down explicitly in terms of Cauchy integrals of known (including the characteristic) functions, if $b$ and $c$ are assumed given. The numerical evaluation of these integrals is harder than the direct numerical integration of (11). This is because the characteristic function that must be used in the exact solution is itself a Cauchy integral. On the other hand, the direct numerical integration of (11) provides a way of avoiding the cumbersome consistency condition as well as the exact characteristic function as it will be explained in the sequel.

\section{Numerical Computation}

We first normalize the interval $(-c, b)$ by the change of variables

$$
\xi / \varepsilon=\delta \omega+\sigma, \quad x / \varepsilon=\delta s+\sigma
$$

where

$$
\delta=\frac{c+b}{2 \varepsilon}, \quad \sigma=\frac{c-b}{2 \varepsilon} .
$$

Eq. (11) becomes

$$
-\beta f F(s) B_{x}(s)+\frac{1}{\pi} \int_{-1}^{1} \frac{B_{x}(\omega)}{\omega-s} d \omega=\frac{1}{C} q^{\infty}\left[1-\frac{p^{\infty}}{q^{\infty}} f F(s)\right], \quad|s|<1
$$

where

$$
F(s)=1+\frac{\Delta f}{f}\left\{1-\left[1-(\delta s+\sigma)^{2}\right] H\left[1-(\delta s+\sigma)^{2}\right]\right\} .
$$


To simplify the numerical computations we will consider $B_{x}(s)$ bounded at 1 and singular at -1 and then require that the intensity of the singularity vanishes at -1 . We may set

$$
B_{x}(s)=\frac{q^{\infty}}{C} w(s) \psi(s),
$$

where $\psi(s)$ is a regular function in the interval $(-1,1)$ and $w(s)$ reflects the nature of the solution at the endpoints [4]:

$$
w(s)=(1+s)^{B}(1-s)^{A}
$$

where

$$
\begin{aligned}
& A=\frac{1}{\pi} \tan ^{-1}[1 / \beta f F(1)], \\
& B=-\frac{1}{\pi} \tan ^{-1}[1 / \beta f F(-1)] .
\end{aligned}
$$

The requirement for bounded $B_{x}(s)$ becomes

$$
\psi(-1)=0 .
$$

Using (17), Eqs. (11) and (12) become

$$
\begin{gathered}
-\beta f F^{\prime}(s) w(s) \psi(s)+\frac{1}{\pi} \int_{-1}^{1} \frac{w(\omega) \psi(\omega)}{\omega-s} d \omega=\frac{p^{\infty}}{q^{\infty}} f F(s), \quad|s|<1 \\
\int_{-1}^{1} w(\omega) \psi(\omega) d \omega=0 .
\end{gathered}
$$

The only numerical quadratures tailored for Cauchy singular integral equations with variable coefficients are, to our knowledge, those developed by Theocaris [5] and Theocaris and Tsamasphyros [6]. In particular, we choose the quadrature which permits the preassignment of the value of the unknown function $\psi(s)$ at the end point -1 . Then, according to [5], [6], (21) can be discretized as

$$
\frac{1}{\pi} \sum_{k=1}^{n} \frac{A_{k}}{1+t_{k}} \frac{\psi\left(t_{k}\right)}{t_{k}-s_{i}}=1-\frac{p^{\infty}}{q^{\infty}} f F\left(s_{i}\right), \quad i=1, \ldots, n+1
$$

where (20) has been taken into consideration. The integration points $t_{k}$ are the roots of the Jacobi polynomial

$$
P_{n}^{(A, 1+B)}\left(t_{k}\right)=0, \quad k=1, \ldots, n
$$

and $A_{k}$ the coefficients of the associated quadrature. The collocation points $s_{i}$ are determined as the roots of some function $G(s)$. This function involves, in general, the computation of Jacobi functions of the second kind, and finding its 
zeros accurately is computationally difficult. If, however

$$
A+B=0,
$$

then $G(s)$ reduces to

$$
\begin{aligned}
G(s)= & {[-\beta f F(s)+\beta f F(1)](1-s)^{A}(1+s)^{-A}(1+s)_{n} P_{n}^{(A, 1-A)}(s) } \\
& -\frac{2}{\sin \pi A} P_{n+1}^{(-A,+1+A)}(s) \frac{1}{(1+s) P_{n}^{(A, 1-A)}}
\end{aligned}
$$

for the present problem, and the points $s_{i}$ are determined from

$$
G\left(s_{i}\right)=0, \quad i=1, \ldots, n+1 .
$$

Moreover, the roots of $G(s)$ alternate with the points $t_{k}$. Fortunately, condition (25) is met if the slip zone extends beyond the interval $(-\varepsilon, \varepsilon)$. This is the case of particular interest, since it is important to know whether a small drop in the coefficient of friction can produce comparatively extensive slip. With this assumption (19) becomes

$$
A=-B=\frac{1}{\pi} \tan ^{-1}\left[\frac{1}{\beta f\left(1+\frac{\Delta f}{f}\right)}\right] .
$$

Using the same quadrature, the disoretized form of $(22)$ is

$$
\sum_{k=1}^{n} \frac{A_{k}}{1+t_{k}} \psi\left(t_{k}\right)=0
$$

Note that (23) and (29) provide $n+2$ equations for the $n+2$ unknowns $\psi\left(t_{k}\right)$, $b$ and $c$. To keep the numerical iterations at a minimum we replace the role of $p^{\infty} / q^{\infty}$ and one of the parameters $b$ or $c$. For instance, we assume $b$ given, make $a$ guess for $c$ and use one equation from the system (26) to solve for $p^{\infty} / q^{\infty}$, which is then eliminated from the remaining equations of the system. We then solve the new system for the values $\psi\left(t_{k}\right)$ and check to see whether (29) is approximately satisfied. If not, we change $c$ and proceed by iteration. Here, we choose $n$ even and use the middle equation of the system (26) to solve for $p^{\infty} / q^{\infty}$.

$$
\begin{gathered}
p^{\infty} / q^{\infty}=\frac{1}{f F^{\prime}(\hat{s})}\left[1-\frac{1}{\pi} \sum_{k=1}^{n} \frac{A_{k}}{1+t_{k}} \frac{1}{t_{k}-\hat{s}} \psi\left(t_{k}\right)\right], \\
\hat{s}=\frac{n}{2}+1 .
\end{gathered}
$$

Using (30), (26) becomes

$$
\begin{gathered}
\frac{1}{\pi} \sum_{k=1}^{n} \frac{A_{k}}{1+t_{k}}\left[\frac{1}{t_{k}-s_{i}}-\frac{1}{t_{k}-\hat{s}} \frac{F\left(s_{i}\right)}{F(\hat{s})}\right] \psi\left(t_{k}\right)=1-\frac{F\left(s_{i}\right)}{F(\hat{s})}, \\
i=1,2, \ldots, \frac{n}{2}, \frac{n}{2}+2, \ldots, n+1
\end{gathered}
$$




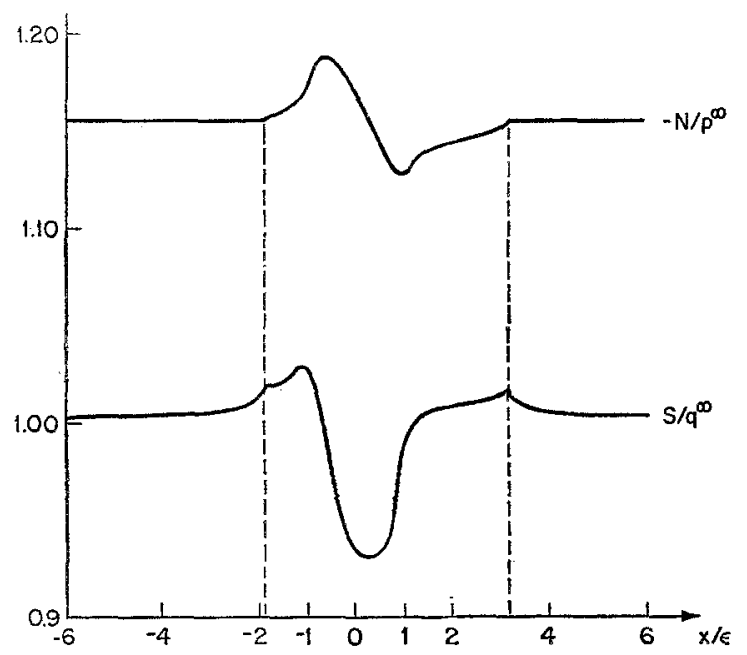

Fig. 3. Normal and shear tractions for $f(x)$ given in Fig. 2

and (29) remains unaltered. Once the values $\psi\left(t_{k}\right)$ are computed, the normal and shear tractions are obtained from (2) and (3). The discretized forms are

$$
\begin{aligned}
S(x) / q^{\infty} & =1-\frac{1}{\pi} \sum_{k=1}^{n} \frac{A_{k}}{1+t_{i}} \frac{\psi\left(t_{i}\right)}{t_{i}-\frac{x-\sigma}{\delta}}, \quad|s|>1 \\
N(x) / q^{\infty} & =\frac{-p^{\infty}}{q^{\infty}}+\beta w(s) \psi(s), \quad|s|<1
\end{aligned}
$$

where $x=\delta s+\sigma$. The shear traction $S(x)$ in the slip zone is obtained from (7). The results are shown in Fig. 3 for a representative case corresponding to

$$
\begin{aligned}
\beta=0.5, \quad f=0.8, \quad \Delta f & =0.08, \quad b=1.9, \quad c=3.14, \\
\frac{p^{\infty}}{q^{\infty}} & =1.155 .
\end{aligned}
$$

It is noted that slip spreads asymmetrically from the origin and that the transitions from slip to stick zones are marked by discontinuous derivatives. The inequalities (9) and (10) are verified. It is also seen that the disturbance produced in the stresses is very localized.

\section{Identical Materials}

As a comparison we also consider the case of identical materials. Then $\beta=0$, $b=c$ and (11) simplifies to

$$
\frac{1}{\pi} \int_{-s}^{c} \frac{B_{x}(\xi)}{\xi-x} d \xi=\frac{x+1}{2 \mu}\left[q^{\infty}-p^{\infty} f(x)\right] .
$$


The solution of (36) with $B_{x}(x)$ bounded is

$$
B_{x}(\xi)=-\frac{(x+1)}{2 \mu \pi} \sqrt{c^{2}-x^{2}} \int_{-c}^{c} \frac{q-p^{\infty} f(\xi)}{\sqrt{c^{2}-\xi^{2}}(\xi-x)} d \xi
$$

provided that

$$
\int_{-c}^{c} \frac{q^{\infty}-p^{\infty} f(x)}{\sqrt{c^{2}-x^{2}}} d x=0
$$

The auxiliary condition (12) is automatically satisfied because of symmetry. Condition (38) can be integrated to yield a relation between $p^{\infty} / q^{\infty}$ and $c$. This relation is

$\left[q^{\infty}-p^{\infty}(f+\Delta f)\right] \pi+p^{\infty} \Delta f\left[\sqrt{\frac{c^{2}}{\varepsilon^{2}}-1}+\left(2-\frac{c^{2}}{\varepsilon^{2}}\right) \sin ^{-1} \frac{\varepsilon}{c}\right]=0$, for $c>\varepsilon$

and

$$
q^{\infty}-p^{\infty}\left(f+\Delta f \frac{c^{2}}{2 \varepsilon^{2}}\right)=0, \text { for } c<\varepsilon
$$

In the limiting case $c=\varepsilon$ both (39) and (40) coincide yielding

$$
p^{\infty} / q^{\infty}=f+\frac{1}{2} \Delta f
$$

The numerical computations presented in the previous section were checked against this result.

\section{Acknowledgements}

We wish to thank Prof. W. Yang for providing a fast subroutine for the computation of the zeros of Eq. (26). Support by the Office of Naval Research through contract N00014-81. $\mathrm{K}-0626$ is gratefully acknowledged.

\section{References}

[1] Rudnicki, J. W., Kanamori, H.: Effects of fault interaction on moment, stress drop, and strain energy release. J. Geophys. Res. 86, 1785 (1918).

[2] Comninou, M.: The interface crack. J. Appl. Mech. 44, 631 (1977).

[3] Tricomi, F. G.: Integral equations. New York: Interscience Publishers, Inc. 1956.

[4] Muskhelishvili, N. I.: Singular integral equations. Groningen: P. Noordhoff 1953.

[5] Theocaris, P. S.: On the numerical solution of Cauchy-type singular integral equations. Serdica Bulg. Math. Publ. 2, 256 (1976).

[6] Theocaris, P. S., Tsamasphyros, G.: Numerical solution of systems of singular integral equations with variable coefficients. Appl. Anal. 9, 37 (1979).

Maria Comninou

Department of Civil Engineering

University of Michigan

Ann Arbor, MI 48109, U.S.A.

\section{J. Dundurs}

Department of Civil Engineering

Northwestern University

Evanston, IL 60201, U.S.A. 